\title{
ASYMPTOTICS OF THE GAUSS HYPERGEOMETRIC FUNCTION WITH LARGE PARAMETERS, II
}

\author{
R. B. PARIS
}

Abstract. We obtain asymptotic expansions by application of the method of steepest descents for the Gauss hypergeometric function

$$
F\left(a+\varepsilon_{1} \lambda, b+\varepsilon_{2} \lambda ; c+\lambda ; z\right)
$$

as $|\lambda| \rightarrow \infty$ when $0<\varepsilon_{1}<1$ and $\varepsilon_{1}>1$ where, without loss of generality, it is supposed that $\varepsilon_{1} \leqslant \varepsilon_{2}$. The resulting expansions are of Poincaré type and break down in the neighbourhood of certain critical points in the $z$-plane. Numerical results illustrating the accuracy of the different expansions are given.

Mathematics subject classification (2010): Primary 33C05, 34E05, 41A60.

Keywords and phrases: Hypergeometric functions, asymptotic expansion, large parameters.

\section{REFERENCES}

[1] F. W. J. Olver, D. W. Lozier, R. F. Boisvert and C. W. Clark (eds.), NiST Handbook of Mathematical Functions, Cambridge University Press, Cambridge, 2010.

[2] R. B. PARIS, Asymptotics of the Gauss hypergeometric function with large parameters, I, J. Classical Anal. 2, 2 (2013), 183-203.

[3] G. N. Watson, Asymptotic expansions of hypergeometric functions, Trans. Cambridge Philos. Soc. 22 (1918), 277-308. 